\title{
Systematic Exploration of Informatics Systems
}

\author{
Peer Stechert \\ Universität Siegen, FB12 DIE, Hölderlinstr. 3, 57076, Siegen, Germany, \\ stechert@die.informatik.uni-siegen.de
}

\section{Summary}

Our research is part of a broader research study towards an education model for understanding of informatics systems at upper secondary level. There are three characteristics specific to informatics systems: their observable behaviour, their internal structure, and implementation aspects. Informatics systems represent networked fundamental ideas of informatics. Thus, networking these concepts within a mental model is essential to understand informatics systems. Observation exercises and systematic exploration have been identified as a means to overcome the cognitive barrier build by complex informatics systems.

Analysis of international curricula and recommendations has shown a lack of strategies to understand the behaviour of informatics systems based on informatics concepts. Thus, we created a sequence of steps to explore an informatics system systematically. Main objective was to make students cope with informatics systems in a conscious way. Hypotheses about intended behaviour of the system avoid trialand-error approaches. We decided to substantiate the systematic exploration with learning theory on problem orientation, educational experiments, and inclusion of different cognitive processes. The steps of the systematic exploration are:

1. Answer questions about the intended behaviour of a given informatics system (remember; factual knowledge),

2. Outline the procedure for conducting the experiment (understanding; procedural),

3. Document the data observed (apply; factual knowledge),

4. Examine data and identify informatics concepts, e.g., access control (analyze; conceptual knowledge),

5. Establish a set of special cases to be tested and evaluate them (evaluate; conceptual knowledge),

6. Create a series of hypotheses for using the informatics system in a realworld or more complex scenario (create; factual knowledge). 
In a first field study we conducted a unit on strategies for understanding of informatics systems. In the informatics course at upper secondary level, 23 students at age 17 have taken part. There have been 13 lessons; three lessons per week. The systematic exploration had to be refined according to a formative evaluation including test, a questionnaire of acceptance by the learner, and an interview with the supervising teacher. One difficulty for students was to combine views on behaviour and internal structure. Searching for the reasons of these difficulties, we see two main aspects: on the one hand, students have no experience with exercises as systematic exploration, so it has to be integrated into the curriculum in more detail and by stating the relevance more precisely. On the other hand, some difficulties could also be caused by typical cognitive barriers and misconceptions of objectoriented modelling. To get more insights, the strategy was investigated applying the Think Aloud method. There are two kinds of results: Firstly, we identified formal problems in the description of the systematic exploration, e.g. terms were unclear, and some focal points of the systematic exploration were not understood. Secondly, we got insights about students' methodologies while they explored an informatics system.

As a last step of the development and refinement cycle, systematic exploration of informatics systems was part of a second curriculum intervention, where the students had to explore two systems given two different schemata: First, the refined, but still abstract steps as described above were given. For a further systematic exploration, a contextualized variation was given. We observed different methodologies: A first group divided the GUI into different parts, which belong together, e.g. belonging to the roles administrator, user, and guest. They described them from the left to the right according to the reading direction. A second group sorted parts of the GUI by programming elements as labels, text fields, and buttons. However, there was anecdotic evidence that the new introduction of the methodology into the classroom, which put more emphasis on the necessity of hypotheses, was successful. During the description of relations between observable elements of the GUI, a student asked: "When can we try out, whether our conjecture holds?" So, the students took making hypotheses more serious, which is necessary for experiments and avoids trial-anderror approaches. 